\title{
Avaliação Preliminar da Qualidade da Madeira de Tectona grandis L. f. Através da Tomografia de Impulso
}

\author{
Juliana Bernardo Amodei ${ }^{1}$, Bruna Roque Ugulino de Oliveira ${ }^{1}$, \\ Monique de Moura Gurgel ${ }^{1}$, Alexandre Monteiro de Carvalho ${ }^{1}$, \\ Reginaldo Antônio Medeiros ${ }^{2}$, João Vicente de Figueiredo Latorraca ${ }^{1}$
}

${ }^{1}$ Universidade Federal Rural do Rio de Janeiro - UFRRJ

${ }^{2}$ Instituto Federal de Educação, Ciência e Tecnologia do Mato Grosso - IFMT

\begin{abstract}
RESUMO
A análise tomográfica de impulso é um método não destrutivo que permite analisar o interior do lenho de árvores pela passagem de ondas mecânicas, tornando possível realizar uma avaliação prévia e detectar a presença de defeitos na madeira. Utilizou-se o tomógrafo de impulso ARBOTOM para analisar o lenho de árvores de Tectona grandis, localizadas em Cáceres, MT. Os resultados possibilitaram detectar a presença de zonas distintas no lenho através da variação da velocidade de ondas mecânicas indicada por variadas zonas de cores reveladas na imagem tomográfica. Possivelmente, essas diferenças de cores são atribuídas às variações da densidade e umidade da madeira.
\end{abstract}

Palavras-chave: métodos não destrutivos, densidade da madeira, tomografia, Tectona grandis.

\section{Preliminary Assessment of Wood Quality of Tectona grandis L. f. by Pulse Tomography}

\begin{abstract}
Impulse tomography analysis is a non-destructive method that enables the visualization of the interior of the wood of trees through the passage of mechanical waves, making it possible to perform a preliminary examination and notice the presence of defects in the wood. ARBOTOM impulse tomography was used to analyze the wood of trees of Tectona grandis, located in Caceres, State of Mato Grosso, Brazil. It was possible to detect the presence of distinct zones in the wood by varying the speed of the wave mechanics indicated by different colored areas in tomography image. Possibly, these color differences are attributed to variations in wood density and moisture content.
\end{abstract}

Keywords: non-destructive method, wood density, tomography, Tectona grandis. 


\section{INTRODUÇÃO}

A Tectona grandis L.f., conhecida popularmente como teca, é uma espécie arbórea que apresenta alto valor comercial. A importância e a valorização da teca se devem às propriedades físico-mecânicas desejáveis da madeira, como durabilidade, estabilidade dimensional, facilidade de prétratamento, resistência natural ao ataque de fungos, insetos, pragas e brocas. Além disso, o desenho e a cor também são aspectos qualitativos importantes que tornam a teca a madeira de folhosa mais valorizada no mundo (Vieira et al., 2002).

Um dos grandes avanços obtidos nos últimos anos na caracterização de materiais é a aplicação de técnicas não destrutivas. Os ensaios não destrutivos são aqueles realizados em materiais para verificar a existência, ou não, de descontinuidades ou defeitos por meio de princípios físicos definidos, sem alterar suas características físicas, químicas, mecânicas ou dimensionais e sem interferir em seu uso (Associação..., 2006).

Atualmente, há uma crescente utilização de técnicas que utilizam informações fornecidas pela passagem de ondas para a análise da qualidade da madeira. Diversos tipos de energia podem ser utilizados como som, ultrassom, eletricidade, raios $\gamma$ e raios X (Nicolotti et al., 2003; Wang \& Allison, 2008).

Nesse contexto, a tomografia de impulso se destaca por ser um método não destrutivo que permite a avaliação do interior do lenho de árvores pela passagem de ondas mecânicas, sendo possível analisar a presença de defeitos (fungos, ataques de insetos, rachaduras, nós, etc.) que alteram as propriedades físicas, químicas e mecânicas da madeira. Seu funcionamento consiste na reconstrução de seções transversais de objetos por meio de informações fornecidas pela passagem de ondas provenientes da superfície (Rinntech, 2005).

O tomógrafo de impulso é composto por sensores que são fixados nas seções transversais das árvores. Ao receberem pancadas de martelo, tais sensores produzem ondas mecânicas, onde o tempo de percurso e a velocidade entre sensores são medidos pelo software do equipamento. Um gráfico de velocidade das ondas mecânicas é gerado, também conhecido como imagem tomográfica. Através das imagens geradas, podem ser obtidas diversas informações como porcentagem de cerne/ alburno, madeira de reação, presença de nós, defeitos internos, podridão do lenho, entre outras (Rinntech, 2005).

A técnica encontra aplicação na indústria madeireira, permitindo a visualização do interior das toras, otimizando a localização e a orientação do corte de tábuas. No setor elétrico e de telefonia, possibilita a visualização do interior de postes de madeira a fim de verificar a presença de falhas e assim avaliar a integridade física e a resistência mecânica destes. Órgãos governamentais e de proteção do meio ambiente também fazem uso da tomografia no diagnóstico de possíveis doenças e/ou mecanismos de degradação.

O presente estudo teve como objetivo analisar o interior do lenho de árvores de Tectona grandis L. f., utilizando o tomógrafo de impulso ARBOTOM para uma avaliação preliminar da qualidade da madeira.

\section{MATERIAL E MÉTODOS}

Foram selecionadas 6 árvores, aleatoriamente, de um povoamento de Tectona grandis L.f. com 11 anos de idade, localizado no campus do Instituto Federal de Educação, Ciência e Tecnologia de Mato Grosso (IFMT), no município de Cáceres, MT ( $16^{\circ} 11^{\prime} 42^{\prime \prime} \mathrm{W}$ e $57^{\circ} 40^{\prime} 51^{\prime \prime} \mathrm{S}, 117 \mathrm{~m}$ de altitude).

Para a realização do exame de tomografia, foi utilizado o tomógrafo de impulso ARBOTOM. Inicialmente, mediu-se a circunferência na altura do DAP (a 1,30 $\mathrm{m}$ do solo) e dividiu-se pelo número de sensores $(n=8)$ para que estes fossem fixados equidistantemente na seção transversal da árvore analisada. Os condutores metálicos foram fixados na árvore para sustentação dos oito sensores de impulsos frontais (Figura 1). Em cada sensor, foram produzidas ondas de impacto através de pancadas de martelete. Essas ondas mecânicas percorreram o lenho até alcançarem os demais sensores e, assim, gerar os tempos de propagação da onda utilizados no cálculo de sua velocidade.

As velocidades das ondas entre os sensores foram calculadas automaticamente pelo software ARBOTOM através da seguinte Equação 1.

$\mathrm{V}=\mathrm{D} / \mathrm{T}$

onde: $\mathrm{V}=$ Velocidade da onda mecânica $(\mathrm{m} / \mathrm{s})$; $\mathrm{D}=$ distância percorrida pela onda $(\mathrm{m}) ; \mathrm{T}=$ tempo 

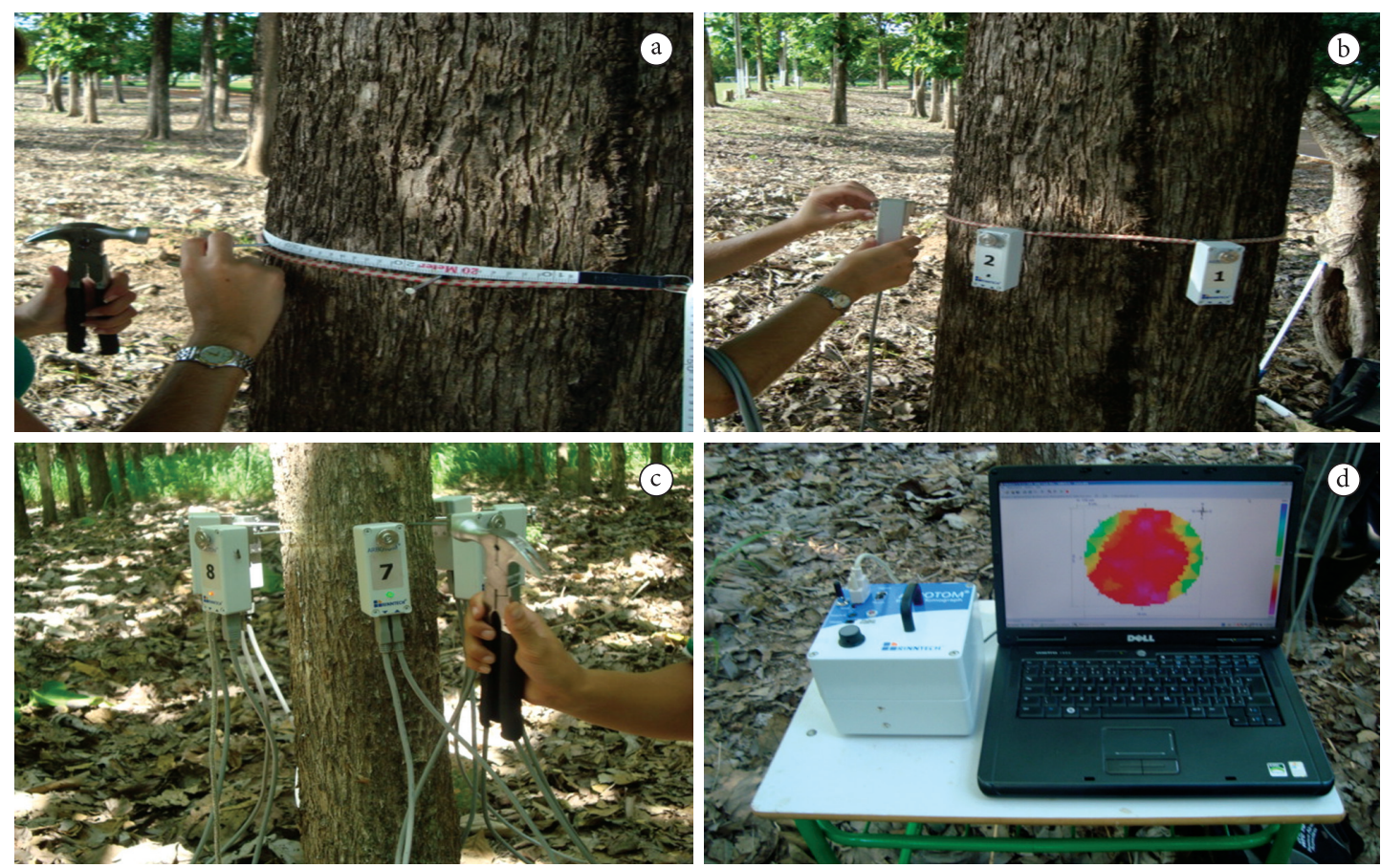

Figura 1. Uso do tomógrafo para obtenção das imagens tomográficas. a) Fixação dos condutores metálicos; b) Distribuição dos sensores; c) Pancadas de martelete para produção de ondas mecânicas; d) Imagem tomográfica. Figure 1. Use tomography to obtain tomographics images. a) Fixing of metal conductors; b) Distribution of sensors; c) Production of mechanical waves; d) Tomographic image.

utilizado para a onda percorrer determinada distância (s).

Em seguida, foi produzido um gráfico de velocidade de onda da seção transversal (imagem tomográfica) de cada árvore.

As variações da coloração nas imagens permitiram identificar as características distintas da madeira, geradas pelas diferentes velocidades de propagação de onda dentro do lenho.

\section{RESULTADOS E DISCUSSÃO}

A técnica de tomografia de impulso utilizada nas árvores de Tectona grandis L.f. gerou as imagens tomográficas apresentadas na Figura 2.

A variação da velocidade de propagação da onda mecânica gerada pelo software do tomógrafo promoveu uma variação na coloração das imagens tomográficas.

De acordo com a escala, observa-se que as porções com coloração azul apresentaram maiores valores de velocidade de onda e as porções com coloração rosa, menores valores de velocidade de onda. Além disso, também foi possível identificar que cada imagem possui um padrão específico na variação das cores relacionadas às velocidades, o que dificulta a comparação entre as imagens.

A velocidade da onda está altamente relacionada com a densidade da área transversal da árvore (Rinntech, 2005). De acordo com Matos (1997), a propagação das ondas no sentido transversal às fibras encontra o diâmetro do lume como barreira e estas desviam, acarretando um aumento acentuado no tempo do percurso, reduzindo significativamente a velocidade das ondas.

A grande variação das velocidades encontradas para as árvores em estudo de mesma idade e provenientes de mesmo povoamento podem ser atribuído às variações anatômicas, físicas e químicas encontradas entre as árvores e até mesmo dentro da mesma árvore. Existem diferentes tipos de madeira no tronco das árvores, relacionados com os estágios de formação do lenho, denominados lenho juvenil (menos denso), de transição e adulto (mais denso). As diferenças particulares entre as espécies precisam 


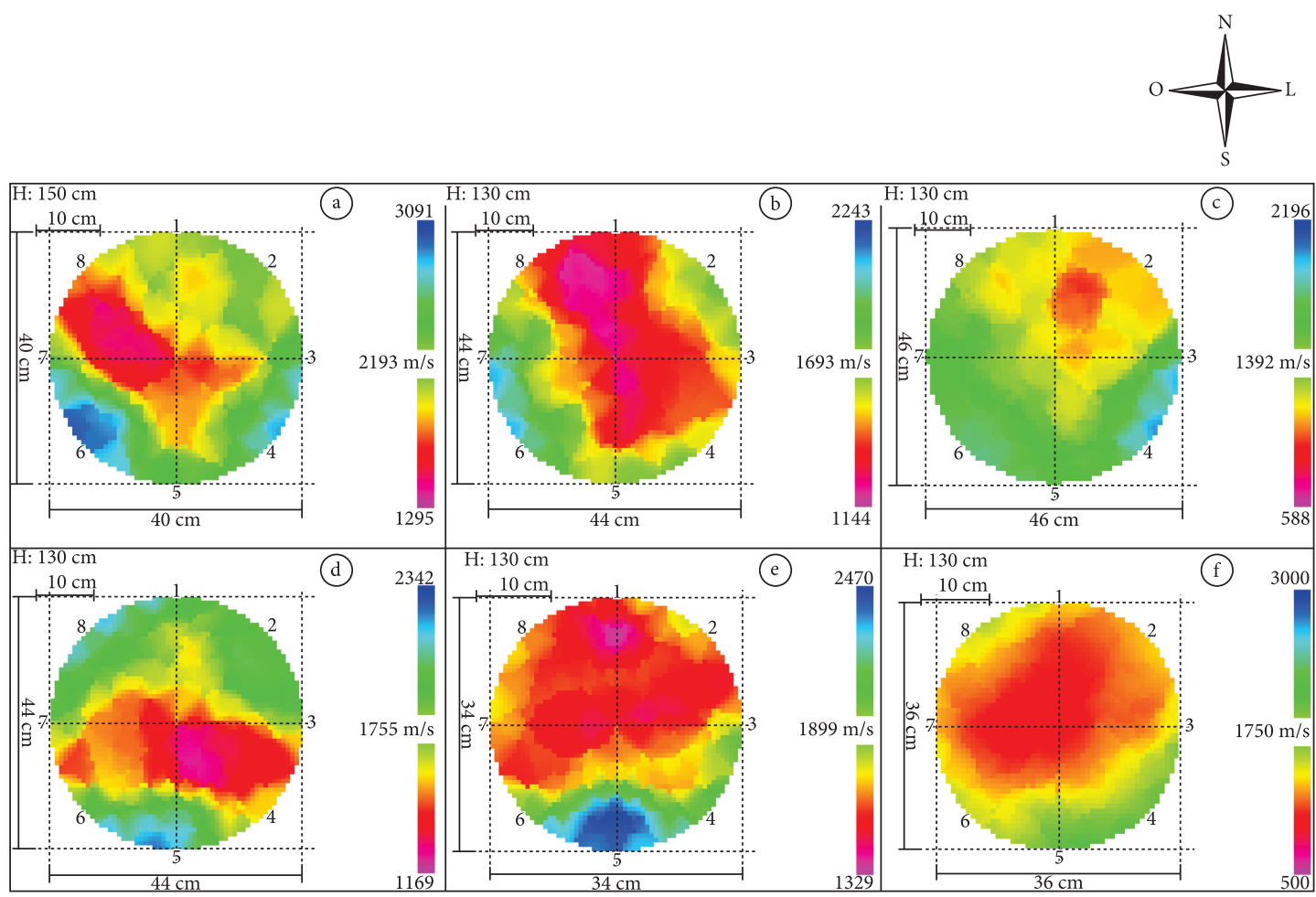

Figura 2. Imagens tomográficas das seções transversais da madeira de Tectona grandis.

Figure 2. Tomography images of cross section of Tectona grandis wood.

ser levadas em conta (Rinntech, 2005). Além disso, pode também ser atribuído a influências externas (vento, chuva, ruídos elevados) e influências inerentes ao equipamento, como qualidade da pancada (diferentes intensidades de batidas nos sensores), fixação dos conectores metálicos.

$\mathrm{Na}$ maioria das imagens (Figuras 2a, 2b, 2d, 2e e 2f), foi possível observar que as porções próximas ao centro apresentaram baixos valores de velocidade de onda (coloração vermelho-rosa), o que se pode inferir que a região próxima à medula é constituída de lenho juvenil, caracterizado por apresentar propriedades físico-mecânicas, como baixa densidade e consequentemente baixa velocidade de propagação de ondas mecânicas.

Outro fator importante que afeta a velocidade de onda é o teor de umidade da madeira, tendo este também relação com a densidade. Dessa forma, as maiores velocidades de propagação de ondas são geralmente obtidas em madeiras que apresentam maiores densidades e menores teores de água (Carrasco \& Azevedo Júnior, 2003; Oliveira Sales, 2005; Bucur, 2006).
Nas Figuras 2a, 2b, 2c, 2d e 2e, foi possível observar porções de coloração azul nas extremidades das amostras, onde o alto valor de velocidade de onda encontrado se deve, provavelmente, à baixa umidade na madeira nessas regiões. Estas porções, também podem ser decorrentes de defeitos ou ataques de organismos xilófagos.

\section{CONCLUSÕES E PERSPECTIVAS}

Com os resultados encontrados, foi possível obter uma avaliação preliminar qualitativa da madeira, de forma não destrutiva, através do uso do tomógrafo de impulso.

A tomografia de impulso é uma técnica não destrutiva de grande importância para estabelecer parâmetros da qualidade da madeira, porém estudos complementares, como avaliação das propriedades físicas, mecânicas, químicas, deverão ser realizados para se estabelecer critérios e parâmetros de avaliação das árvores e predizer as possibilidades de uso da madeira. 


\section{AGRADECIMENTOS}

Os autores agradecem à Coordenação de Aperfeiçoamento de Pessoal de Nível Superior CAPES e ao Instituto Federal de Educação, Ciência e Tecnologia do Mato Grosso - IFMT, Campus Cáceres.

\section{STATUS DA SUBMISSÃO}

Recebido: $24 / 06 / 2010$

\section{AUTOR(ES) PARA CORRESPONDÊNCIA}

\section{Juliana Bernardo Amodei}

Universidade Federal Rural do Rio de Janeiro, BR 465, Km 07, CEP 23890-000,

Seropédica, RJ, Brasil

e-mail: juliana_floresta@yahoo.com.br

\section{REFERÊNCIAS}

Associação Brasileira de Ensaios Não-Destrutivos e Inspeção. Ensaios Não Destrutivos. [cited 2006 jul. 12]. Available from: http://www.abende.org.br/ensaios_ nao_destrutivos.html>.
Bucur V. Environment modifiers of Wood structural parameters detected with ultrasonic waves. In: Bucur V. Acoustics of Wood. $2^{\text {rd }}$ ed. Berlin, Heidelberg: SpringerVerlag; 2006. chap. 9, p. 242-270.

Carrasco EVM, Azevedo Júnior AP. Avaliação nãodestrutiva de propriedades mecânicas de madeiras através de ultra-som - fundamentos físicos e resultados experimentais. Cerne 2003; 9(2):178-191.

Matos JLM. Estudos sobre a produção de painéis estruturais de lâminas paralelas de Pinus taeda $L$ Curitiba [tese]. Curitiba: Setor de Ciências Agrárias, Universidade Federal do Paraná; 1997.

Nicolotti G, Socco LV, Martinis R, Godio A, Sambuelli L. Application and comparison of three tomographic tecniques for detection of decay in trees. Journal of Arboriculture 2003; 2(29):66-78.

Rinntech. User Manual - Arbotom 3-D Tree Impulse Tomograph, version 1.59 for Microsoft Windows 98, 2000, XP. Heidelberg: Microsoft Window; 2005. 42 p.

Vieira AH, Martins EP, Pequeno PLL, Locatelli M. Aspectos silviculturais da teca em Rondônia. Porto Velho: Embrapa CPAF, Ministério da Agricultura, Pecuária e Abastecimento; 2002. Documentos, n. 68).

Wang X, Allison B. Decay detection in red oak trees using a combination of visual inspection, acoustic testing and resistance micro drilling. Journal of Arboriculture 2008; 34(1):1-14. 